\title{
Convergent gain and loss of genomic islands drive lifestyle changes in plant-associated Pseudomonas
}

\author{
Ryan A. Melnyk ${ }^{1,2} \cdot$ Sarzana S. Hossain ${ }^{1}$ Cara H. Haney $\mathbb{D}^{1}$
}

Received: 10 October 2018 / Revised: 4 January 2019 / Accepted: 31 January 2019 / Published online: 20 February 2019

(c) International Society for Microbial Ecology 2019

\begin{abstract}
Host-associated bacteria can have both beneficial and detrimental effects on host health. While some of the molecular mechanisms that determine these outcomes are known, little is known about the evolutionary histories of pathogenic or mutualistic lifestyles. Using the model plant Arabidopsis, we found that closely related strains within the Pseudomonas fluorescens species complex promote plant growth and occasionally cause disease. To elucidate the genetic basis of the transition between commensalism and pathogenesis, we developed a computational pipeline and identified genomic islands that correlate with outcomes for plant health. One island containing genes for lipopeptide biosynthesis and quorum-sensing is required for pathogenesis. Conservation of the quorum-sensing machinery in this island allows pathogenic strains to eavesdrop on quorum signals in the environment and coordinate pathogenic behavior. We found that genomic loci associated with both pathogenic and commensal lifestyles were convergently gained and lost in multiple lineages through homologous recombination, possibly constituting an early step in the differentiation of pathogenic and commensal lifestyles. Collectively this work provides novel insights into the evolution of commensal and pathogenic lifestyles within a single clade of hostassociated bacteria.
\end{abstract}

\section{Introduction}

Host-adapted bacterial lifestyles range from mutualistic to commensal to pathogenic resulting in positive, neutral, or negative effects on host fitness, respectively [1]. Many of these intimate associations are the product of millions of years of co-evolution, resulting in a complex molecular dialogue between host and bacteria [2, 3]. In contrast, horizontal gene transfer (HGT) can lead to rapid lifestyle transitions in host-associated bacteria through the gain and loss of virulence genes [4-6]. For example, the acquisition and loss of pathogenicity islands plays a key role in the

Supplementary information The online version of this article (https:// doi.org/10.1038/s41396-019-0372-5) contains supplementary material, which is available to authorized users.

Cara H. Haney

cara.haney@msl.ubc.ca

1 Department of Microbiology and Immunology, The University of British Columbia, Vancouver, BC, Canada

2 Present address: Department of Plant Biology, University of California, Davis, Davis, CA, USA emergence of enteropathogenic Escherichia coli strains from commensal lineages and vice versa [5]. Similarly, a virulence plasmid transforms beneficial plant-associated Rhodococcus strains into pathogens, while strains without the plasmid revert to commensalism [4]. It is unclear if reversibility of lifestyles is common in other bacteria, or if acquisition of pathogenicity genes drives loss of genomic features associated with commensalism (or vice versa).

To further examine how changes in genome content might influence bacterial lifestyle, we focused on the Pseudomonas fluorescens (Pfl) species complex, which contains both commensal strains and pathogens [7-13]. Pfl strains are enriched in close proximity to plant roots (the "rhizosphere") relative to surrounding soil in diverse plants including the model plant Arabidopsis thaliana [9, 14, 15]. Single Pfl strains benefit Arabidopsis health by promoting lateral root formation, protecting against pathogens, and modulating plant immunity $[9,16]$. However, some Pfl strains cause diseases such as tomato pith necrosis [17] and rice sheath rot [18]. Thus, we used the Pfl species complex in association with Arabidopsis to understand how strains shift along the symbiosis spectrum from pathogenic to commensal and how lifestyle might influence genome evolution. 
Here we show that among several closely related Pseudomonas strains sharing $>99 \%$ 16S rRNA identity, gain and loss of multiple genomic islands through homologous recombination can drive the transition from pathogenesis to commensalism. Using a novel high-throughput comparative genomics pipeline followed by reverse genetics, we found two unique sets of genomic features associated with predicted pathogenic and commensal strains. Evolutionary reconstruction indicates that gain and loss of these genomic features occurred multiple times, and that the gains and losses were mediated by homologous recombination of regions flanking conserved insertion sites. Collectively, this work implicates interactions between homologous recombination and HGT as the primary drivers of lifestyle transitions in the rhizosphere.

\section{Materials and methods}

\section{Bacterial cultivation and quorum biosensor assay}

All Pseudomonas wild-type and mutant strains in this study were routinely cultured in LB (lysogeny broth) media at $28{ }^{\circ} \mathrm{C}$. Detailed information on all strains used in this study can be found in Table S1. The quorum-sensing biosensor assay was carried out by restreaking single colonies of wild-type and mutant strains on LB with $1.5 \%$ agar next to a streak of Chromobacterium violaceaum CV026, then incubating overnight at $28^{\circ} \mathrm{C}[19,20]$. For selected strains in the Pseudomonas brassicacearum clade (as well as the $\Delta l u x I_{\mathrm{LPQ}} \mathrm{N} 2 \mathrm{C} 3$ mutant), we also performed a quantitative assessment of violacein production as previously described [21]. Briefly, each strain of interest was streaked three times next to a streak of $C$. violaceum CV026 on LB agar and incubated overnight. The next day, each CV026 streak was scraped off the plate using an inoculation loop and resuspended in $1 \mathrm{~mL} 10 \mathrm{mM} \mathrm{MgSO} 4$. One hundred and fifty microliters of the suspension was diluted into $10 \mathrm{mM} \mathrm{MgSO}_{4}$ prior to an absorbance reading at $764 \mathrm{~nm}$ to measure cell density. To normalize cell density, $764 \mathrm{~nm}$ was used because violacein absorbs near the $600 \mathrm{~nm}$ wavelength normally used for optical density (OD). The remaining suspension was centrifuged for $1 \mathrm{~min}$ at $6000 \mathrm{rcf}$, the supernatant was removed, and the pellet was resuspended in $1 \mathrm{~mL}$ dimethyl sulfoxide and incubated for $30 \mathrm{~min}$ at room temperature. After incubation, samples were centrifuged at 15,000 rcf for 15 mins. Five hundred microliters of the supernatant was mixed with $500 \mu \mathrm{L} 10 \mathrm{mM} \mathrm{MgSO}_{4}$ and the absorbance was measured at violacein's absorbance peak of $585 \mathrm{~nm}$. Relative violacein production was reported as the ratio of the $\mathrm{OD}_{585} / \mathrm{OD}_{764}$ measurements to account for differences in cell density.

\section{Gnotobiotic Arabidopsis root inoculation assays}

A. thaliana seeds of the Col-0 ecotype were sterilized with a 3-min $70 \%$ ethanol treatment followed by $50 \%$ bleach for $10 \mathrm{~min}$. Seeds were then washed thrice in sterile water and stored at $4{ }^{\circ} \mathrm{C}$ in the dark for $48 \mathrm{~h}$ prior to sowing on square plates with solid half-strength Murashige and Skoog (MS) media containing no sucrose and $1 \%$ PhytoAgar. Seeds were sowed on the surface and the plates were sealed with MicroPore tape and stored vertically so that roots grew along the surface of the media. Seedlings were grown at $23^{\circ} \mathrm{C}$ under $100 \mu \mathrm{E}$ cool white fluorescent lights and a $16 \mathrm{~h}$ light $/ 8 \mathrm{~h}$ dark cycle. Roots were inoculated 5-7 days after sowing. Bacterial inocula were prepared by streaking out a freezer stock, then picking single colonies into an overnight $5 \mathrm{~mL}$ culture of LB. Aliquots from the overnight culture were centrifuged for $1 \mathrm{~min}$ at $6000 \times g$, then resuspended in $10 \mathrm{mM} \mathrm{MgSO}_{4}$. The resuspensions were then diluted to an OD measured at $600 \mathrm{~nm}$ of 0.1 , followed by another 100 fold dilution into $10 \mathrm{mM} \mathrm{MgSO}_{4}$. A volume of $5 \mu \mathrm{L}$ of this final dilution was used to inoculate along the primary root of each seedling. Plates were resealed with Micropore tape and returned to the growth chamber for 7-8 days, after which lateral roots were counted, primary roots were measured using scanned plates and ImageJ, and/or whole seedlings were weighed depending on the experiment. Boxplots for all quantitative measurements represent quartiles of the data with outliers discarded. Significance tests of mean values (with outliers included) were carried out using a two-sided unpaired $T$-test as implemented by the "stats. ttest_ind" function in SciPy (https://www.scipy.org). Lowercase letters are used on the boxplots to denote statistical significance below a threshold of $p=0.01$.

\section{Gnotobiotic seed treatment assay for non- Arabidopsis plants}

All seeds were sterilized with a 3-min $70 \%$ ethanol treatment followed by $50 \%$ bleach for $10 \mathrm{~min}$. Twenty to forty seeds were sown onto $80 \mathrm{~mL}$ of $1 / 2$ strength MS media with $1 \%$ Phytoagar in cylindrical plant growth containers. One milliliter of overnight cultures of $\mathrm{N} 2 \mathrm{C} 3$ and $\mathrm{N} 2 \mathrm{E} 3$ in $\mathrm{LB}$ were centrifuged and washed three times in $10 \mathrm{mM} \mathrm{MgSO}$ before being diluted to an $\mathrm{OD}_{600}$ of 0.1. One milliliter of the bacterial suspension was dripped directly onto seeds immediately after sowing. Containers were returned to the growth chamber before being imaged at 8 or 15 days after inoculation.

\section{Gene deletions in Pseudomonas sp. N2C3}

Deletion mutants were created using a double-crossover methodology common for making deletions in Gram-negative 
bacteria (e.g. [22]). Briefly, fragments of 800-900 bp flanking the gene of interest were amplified using PCR from genomic DNA. External primers had $5^{\prime}$ extensions adding a restriction site, while internal primers were designed to be in-frame with the gene of interest and had either another $5^{\prime}$ restriction site $(\Delta l u x I)$ or a 21 -bp linker $(\Delta \mathrm{SYP}, \Delta \mathrm{SYR}$, and $\Delta l u x R)$. The fragments flanking the luxI gene were assembled using threeway ligation into the pNPTS138 vector (MRK Alley, unpublished data) while the fragments flanking the remaining genes were assembled using overlap extension PCR into the pEXG2 suicide vector[23]. Primers for deletions can be found in Table S2.

Plasmids were transformed into chemically competent aliquots of the diaminopimelic acid (DAP) auxotroph $E$. coli WM3064 and plated on LB containing $0.3 \mathrm{mM}$ DAP and the appropriate antibiotic $(10 \mu \mathrm{g} / \mathrm{mL}$ gentamycin for pEXG2-derived vectors and $50 \mu \mathrm{g} / \mathrm{mL}$ kanamycin for pNPTS138-derived vectors). Individual colonies were picked into overnight cultures in LB with antibiotic and DAP. One milliliter of the transformed WM3064 overnight culture was washed $3 x$ in LB to remove antibiotics before being centrifuged at $6000 \times g$ with $1 \mathrm{~mL}$ of overnight culture of wild-type Pseudomonas sp. N2C3. The supernatant was decanted and the combined bacteria were resuspended in the liquid that remained $(\sim 30 \mu \mathrm{L})$. This mixed bacterial suspension was spotted onto an LB plate and incubated at $28^{\circ} \mathrm{C}$ for $4-6 \mathrm{~h}$. After $4-6 \mathrm{~h}$, the spot was restreaked directly onto $\mathrm{LB}$ containing the appropriate antibiotic to isolate N2C3 transconjugants. We then restreaked strains again on the appropriate antibiotic before restreaking on nosalt LB with $10 \%$ sucrose for counterselection against the integrated plasmid.

We began our work using the pNPTS138 suicide vector, which confers kanamycin resistance and sucrose sensitivity. However, we found that all Pseudomonas clones with an integrated pNPTS138 vector were still sucrose-resistant. Thus, for the $\Delta$ luxI mutant, we screened roughly $\sim 50$ colonies to identify spontaneous mutants that lost the plasmid. This is consistent with a previous observation that the $s a c B$ gene was insufficiently expressed in Pseudomonas aeruginosa for lethality on sucrose-containing media, which led to the generation of pEXG2, which has increased expression of $s a c B$ [23]. Therefore, we used $\mathrm{pEXG} 2$ for all future deletions for stronger counterselection against $s a c B$.

Once antibiotic-sensitive strains were recovered, colony PCR was performed using the upstream and downstream primers to distinguish wild-type revertants from deletion mutants. Deletion mutants were restreaked for purity, patched again on antibiotic-containing media to confirm plasmid loss, and verified again using colony PCR before being stored as a freezer stock. The $\Delta \mathrm{SYR} \Delta \mathrm{SYP}$ double-mutant strain was constructed by introducing the $\triangle \mathrm{SYR}$ deletion construct into the previously constructed $\triangle \mathrm{SYP}$ mutant.

\section{$16 \mathrm{~S}$ similarity}

We extracted full-length 16S rRNA gene sequences from the genomes of the strains in the $P$. brassicacearum clade. Using USEARCH to cluster $16 \mathrm{~S}$ sequences at a threshold of 97\% identity yielded a single cluster [24]. Increasing the USEARCH threshold to $99 \%$ yielded a second cluster containing two strains with more divergent $16 \mathrm{~S}$ sequences (Pseudomonas sp. P97-38 and Pseudomonas chlororaphis GCA001023535), but the majority of sequences still mapped to a single cluster. This suggests that the diversity of this entire clade would be represented by a single operational taxonomic unit (OTU) in amplicon-based sequencing studies.

\section{Computational methods}

A full description of the development, benchmarking, and utilization of the PyParanoid pipeline, as well as details on comparative genomics and phylogenetic methods can be found in the Supplementary Information.

\section{Results}

\section{A pathogen within a plant growth-promoting clade}

To understand the emergence and phylogenetic distribution of plant-associated lifestyles, we focused on a wellcharacterized commensal strain with plant-beneficial activity and asked whether its closest cultured relatives also had beneficial effects on plant hosts. Pseudomonas sp. WCS365 robustly colonizes plant roots [25, 26], promotes growth [9], and protects plants from soil-borne fungal pathogens [27]. Close relatives of WCS365 include an isolate from Arabidopsis (P. brassicacearum NFM421) [28], and isolates from a nitrate-reducing enrichment of groundwater (N2E2 and N2C3) [29, 30] (Fig. 1a). Together, these four strains share nearly identical 16S rRNA sequences ( $>99.4 \%$ identity) and would be grouped into a single OTU in a community profile; however, it is unknown whether all members of this OTU share the beneficial antifungal and plant growth promotion abilities of WCS365.

We tested whether these four closely related strains could promote plant growth. We found that in a gnotobiotic seedling assay where WCS365, NFM421, and N2E2 increased lateral root density and had no significant effect on fresh weight (Fig. 1b, c), N2C3 caused significant stunting of primary root and rosette development (Fig. 1b) and a significant reduction in fresh weight relative to mockinoculated seedlings (Fig. 1c). N2C3 also increased lateral root density, however, whether this is due to increased lateral root initiation or inhibition of primary root 


\section{A}

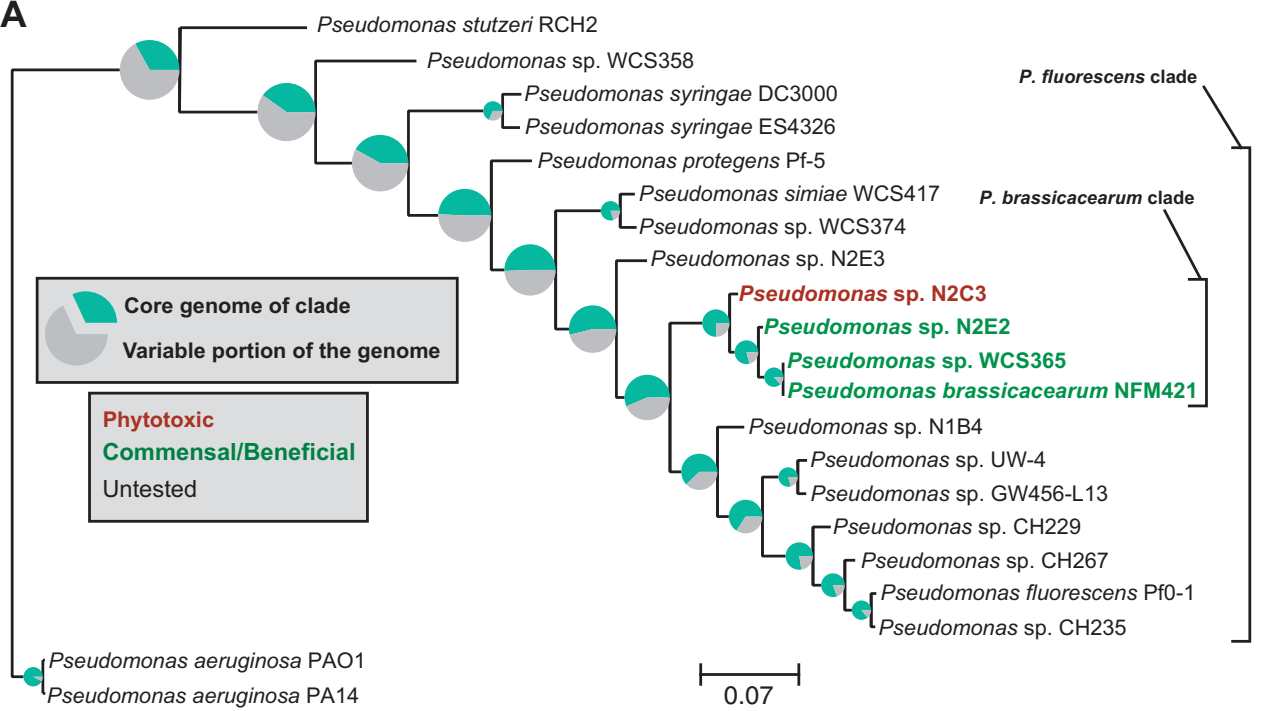

B

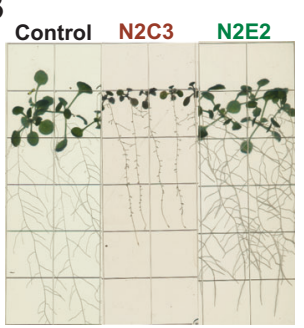

C
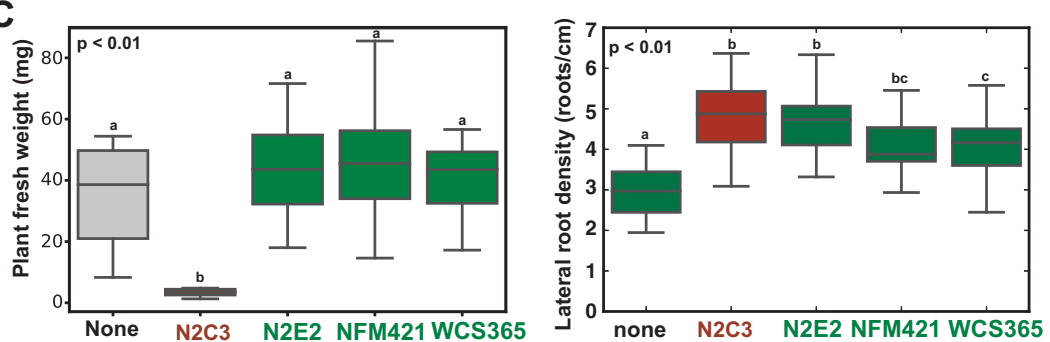

Fig. 1 A pathogen within a clade of beneficial Pseudomonas spp. a A species tree of characterized Pseudomonas strains was generated using 217 conserved housekeeping genes. Strain names are color-coded to reflect plant-associated lifestyles based on their effects on Arabidopsis. Pie charts at each node reflect the proportion of the genome that encodes "core" genes that are present in each member of the clade, showing that there can be large differences in gene content over short evolutionary distances. b Gnotobiotic Arabidopsis seedlings

elongation is unclear (Fig. 1b, c). Additionally, we found that N2C3 killed or stunted plants from the families Brassicaceae (kale, broccoli, and radish) and Papaveroideae (poppy), but had little to no effect on plants in the Solanaceae (tomato and Nicotiana benthamiana) (Figure S1). Thus, unlike its close relatives that promote plant growth, $\mathrm{N} 2 \mathrm{C} 3$ is a broad host range pathogen under laboratory conditions.

\section{A pathogenicity island found in plant-associated Pfl}

We reasoned that by comparing the genomic content of $\mathrm{N} 2 \mathrm{C} 3$ to closely related commensal Pfl strains and pathogenic Pseudomonas syringae strains, we could identify the genetic mechanisms underlying pathogenicity or commensalism within this clade. The large number of sequenced genomes within the genus Pseudomonas made existing homolog detection methods (which scale exponentially) untenable for surveying the pangenome of the inoculated with N2C3, N2E2, or a buffer control. c Fresh weight of seedlings treated with $10 \mathrm{mM} \mathrm{MgSO}$, N2C3, N2E2, NFM421, and WCS365. Lateral root density of seedlings treated with $10 \mathrm{mM}$ $\mathrm{MgSO}_{4}, \mathrm{~N} 2 \mathrm{E} 2, \mathrm{NFM} 421$, and WCS365. Lower-case letters indicate statistically significantly different $(p<0.01,26>n>12)$ means as determined by a two-sided Student's $T$-test for unpaired samples. Boxplots are quartile plots of the observed data. Data shown are from one of two independent experiments performed with similar results

entire genus [31]. Therefore, we sought to develop a method that coupled fast but robust ortholog identification of a reference pangenome with a heuristic approach that generated binary homolog presence-absence data for an arbitrarily large dataset.

In order to identify the genomic features associated with commensalism and pathogenesis, we built a bioinformatics pipeline called PyParanoid to generate the Pseudomonas reference pangenome on large genomic datasets. A detailed description of PyParanoid can be found in the Supplementary Information and an overview is shown in Figure S2. Briefly, PyParanoid uses conventional similarity clustering methods to identify the pangenome of a training dataset that includes phylogenetically diverse reference genomes and strains of experimental interest. The diversity of the training pangenome is then represented as a finite set of amino acid hidden Markov models, which are then used in the second phase to catalog the pangenome content using computational resources that scale linearly (not exponentially) with 
the size of the dataset. The result of this pipeline is presence-absence data for a genome dataset that is not constrained by sampling density or phylogenetic diversity. This heuristic-driven approach enabled us to rapidly assign presence and absence of 24,066 discrete homology groups to 3894 diverse genomes from the diverse Pseudomonas genus, assigning homology group membership to $94.2 \%$ of the 22.6 million protein sequences in our combined database (details in Supplemental Methods and Figure S2). The construction of the Pseudomonas pangenome database was accomplished using reasonable computational resources (roughly $\sim 230$ core-hours on a single workstation). We also benchmarked PyParanoid on a series of test datasets against OrthoFinder2 [32, 33]. PyParanoid was much faster than OrthoFinder2 ( $2.7 \mathrm{~h}$ vs $36.3 \mathrm{~h}$ for a 120 -strain dataset), but sacrificed no accuracy in homolog detection as determined by assessing the capture of a group of known single-copy genes, with both methods yielding a capture rate of $99.8 \%$ (details in Supplemental Methods and Figure S3).

Using the Pseudomonas reference pangenome, we searched for genes that were unique to the pathogenic $\mathrm{N} 2 \mathrm{C} 3$ or its three closely related commensal relatives with plantbeneficial activity. We found that $\mathrm{N} 2 \mathrm{C} 3$ contains a conspicuously large $143-\mathrm{kb}$ island comprising $2.0 \%$ of the $\mathrm{N} 2 \mathrm{C} 3$ genome that is not present in the other strains. The predicted functions of the genes are also consistent with a role in pathogenesis; the island features two adjacent large clusters of non-ribosomal peptide synthetase genes, as well as genes similar to the acyl-homoserine lactone (AHL) quorum-sensing system prevalent in the Proteobacteria, which can play a role in virulence [34] (Fig. 2a). We designated this putative pathogenicity island the LPQ island (lipopeptide/quorum-sensing). These clusters are very similar to genes involved in the production of cyclic lipopeptide pore-forming phytotoxins in $P$. syringae spp. (syringopeptin and syringomycin), which have roles in virulence in many pathovars of $P$. syringae [35-37]. The genomic regions flanking each end of the lipopeptide island in $\mathrm{N} 2 \mathrm{C} 3$ are adjacent to one another in the genome of N2E2 (Fig. 2b) suggesting HGT of the island. HGT has been reported for similar lipopeptide clusters in certain pathovars of $P$. syringae [38].

In order to determine if the LPQ island is necessary for pathogenesis in the Pfl clade, we used reverse genetics to disrupt portions of the LPQ island in $\mathrm{N} 2 \mathrm{C} 3$. We made clean deletion muants of gene clusters predicted to encode syringopeptin $(\triangle \mathrm{SYP}-73 \mathrm{~kb})$ and syringomycin $(\triangle \mathrm{SYR}-39$ $\mathrm{kb}$ ), in addition to a mutant with both clusters deleted $(\triangle \mathrm{SYR} \triangle \mathrm{SYP})$. We found that deletion of either cluster eliminated the $\mathrm{N} 2 \mathrm{C} 3$ pathogenesis phenotype (Fig. 2c). This is consistent with observations that both syringomycin and syringopeptin contribute to virulence in $P$. syringae $\mathrm{B} 301 \mathrm{D}$ [35]. The $\Delta$ SYR $\Delta$ SYP mutant elicited increased lateral root density compared to an untreated control (Fig. 2d). We also generated knockouts of both the AHL synthase $\left(\mathrm{LuxI}_{\mathrm{LPQ}}\right)$ as well as the AHL-binding transcriptional regulator (LuxR $\mathrm{LPQ}_{\mathrm{LP}}$ ). Both the $\triangle \operatorname{luxI}_{\mathrm{LPQ}}$ and $\triangle l u x R_{L P Q}$ mutations abrogated the pathogenic phenotype (Fig. 2e). These genetic results indicate that both lipopeptide biosynthesis and quorum-sensing within the LPQ island are required for the pathogenicity of $\mathrm{N} 2 \mathrm{C} 3$.

Because the LPQ island is necessary for pathogenesis in $\mathrm{N} 2 \mathrm{C} 3$, we speculated that it may serve as a marker for pathogenic behavior in other Pseudomonas strains. We searched the PyParanoid database for other strains with genes contained within the 15 homology groups unique to the lipopeptide island (Table S3). While many of the lipopeptide biosynthesis-associated genes were found in a subset of $P$. syringae strains, the entire set of 15 genes including the quorum-sensing system were found in three other species that contain bona fide plant pathogens (Pseudomonas corrugata, Pseudomonas mediterranea, and Pseudomonas fuscovaginae sensu lato) within the Pfl clade as well as several strains with known antifungal activity (Fig. 2f and Table S3). Genomic and genetic evidence from these three pathogenic species support a role for the LPQ island in pathogenesis in a variety of hosts, suggesting that the mechanism used by $\mathrm{N} 2 \mathrm{C} 3$ to kill Arabidopsis may be conserved in divergent strains throughout the Pfl clade [7, 8, 39-42]. Additionally, it was previously shown that the LPQ island is the source of antifungal cyclic lipopeptides in two other strains (DF41 and in5) [43, 44]. Moreover, in $P$. corrugata and $P$. mediterranea, quorum-sensing is found to have a role in modulating lipopeptide production, although it does not seem to affect lipopeptide production in DF41 $[41,42,45]$. Collectively these data indicate that the LPQ island serves as a marker for plant-pathogenic behavior and/ or antifungal activity in diverse Pseudomonas spp.

To determine if the presence of the island predicted pathogenesis, we tested 14 additional isolates including 5 that contain the island and 9 that do not. Of the 5 new isolates that contain the island, 4 ( $P$. mediterranea CFBP 5447, $P$. corrugata DSM 7228 , and $P$. fuscovaginae-like strains SE-1 and IRRI 6609) were originally isolated from diseased plant tissues and are capable of pathogenesis, whereas DF41 was isolated from canola root tips and is a commensal strain with antifungal activity (Table S1). The remaining 9 new isolates that do not contain the island were a mix of plant-associated and environmental isolates. The 4 pathogenic isolates containing the island inhibited Arabidopsis to a similar degree as N2C3 (Fig. 2f, g). On the other hand, DF41 did not inhibit Arabidopsis growth (Fig. 2f, g) nor did any of the 9 new isolates that do not contain the island. The presence/absence of the LPQ island predicted pathogenic behavior in 17/18 (94\%) of total wild-type isolates tested suggesting it may serve as a genetic marker for 
A

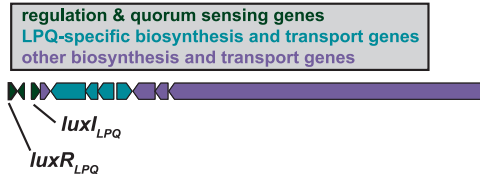

B

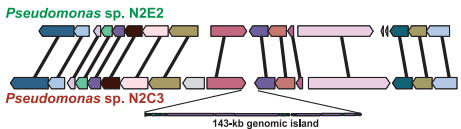

C

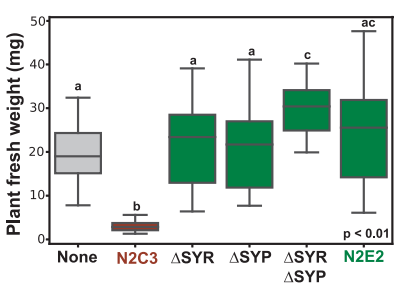

syringopeptin synthetase operon (SYP)

GODOD UOS

syringomycin synthetase operon (SYR)

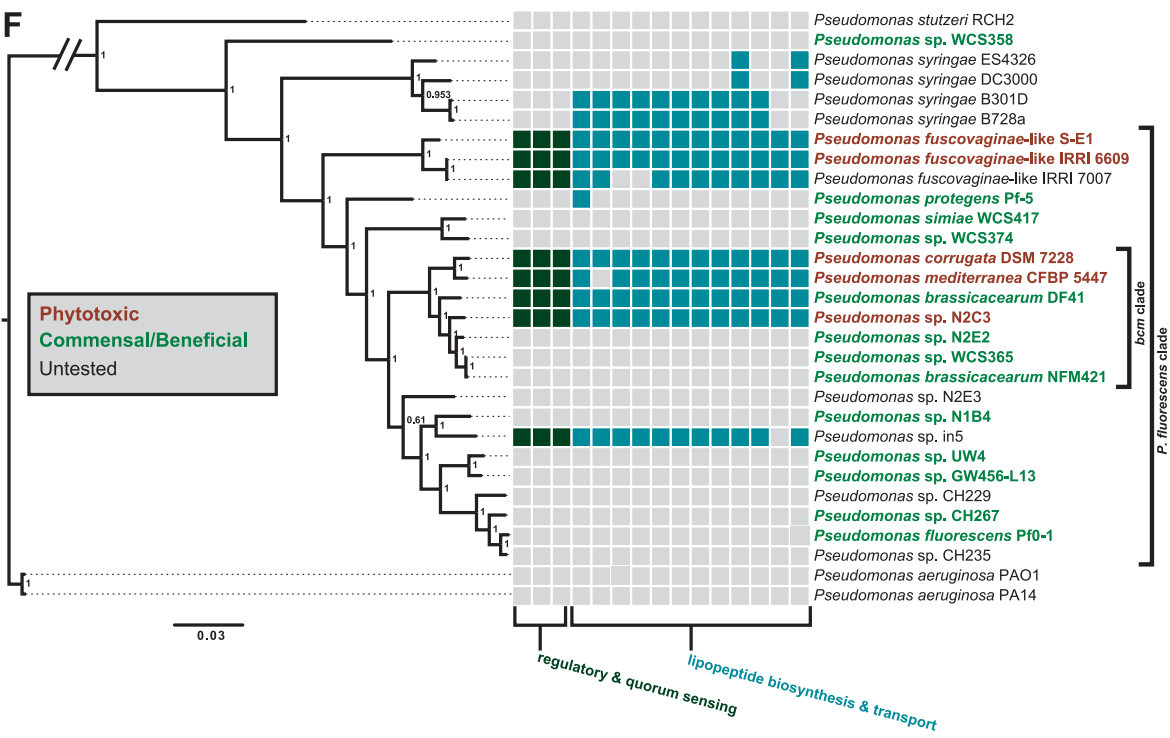

G
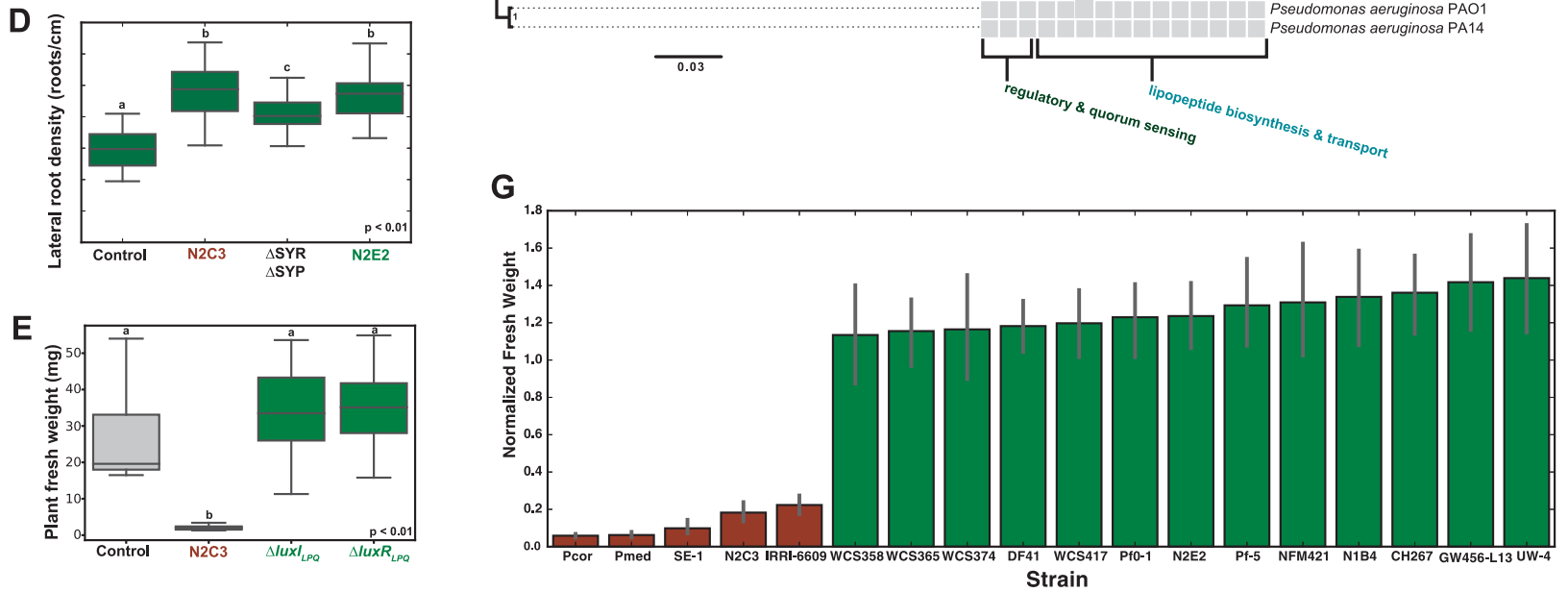

Fig. 2 A genomic island necessary for pathogenesis. a A large genomic island encoding lipopeptide biosynthesis and quorum-sensing genes (the LPQ island) is present in $\mathrm{N} 2 \mathrm{C} 3$ but not in beneficial strains such as N2E2. b Diagram of the genomic island showing putative functions and locations of quorum-sensing genes (luxI $I_{L P Q}$-acylhomoserine lactone (AHL) synthase, $\operatorname{lux} R_{L P Q}-\mathrm{AHL}-$ binding transcriptional regulator) and the lipopeptide biosynthesis clusters (syringomycin and syringopeptin). c Fresh weight of Arabidopsis treated with $10 \mathrm{mM} \mathrm{MgSO}_{4}$, wild-type $\mathrm{N} 2 \mathrm{C} 3$, wild-type N2E2, N2C3 with a syringomycin cluster deletion $(\triangle \mathrm{SYR})$, a syringopeptin cluster deletion $(\triangle \mathrm{SYP})$, and a syringomycin and syringopeptin double deletion $(\triangle \mathrm{SYR} \triangle \mathrm{SYP})$. Lowercase letters indicate statistically significant $(p<$ $0.01,20>n>15$ ) differences as determined by a two-sided unpaired Student's $T$-test. Boxplots are quartile plots of the observed data. Data shown are from one of two independent experiments performed with similar results. d Fresh weight of Arabidopsis treated with $10 \mathrm{mM}$ $\mathrm{MgSO}_{4}$, wild-type $\mathrm{N} 2 \mathrm{C} 3, \mathrm{~N} 2 \mathrm{C} 3$ with an $\mathrm{AHL}$ synthase deletion,

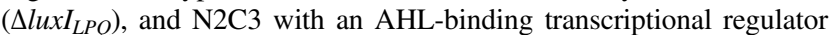
deletion $\left(\triangle l u x R_{L P Q}\right)$. Lowercase letters indicate statistically significant $(p<0.01,11>n>5)$ differences as determined by a two-sided unpaired Student's $T$-test. Data shown are from one of two

independent experiments performed with similar results. Boxplots are quartile plots of the observed data. e Lateral root density of seedlings treated with $10 \mathrm{mM} \mathrm{MgSO}_{4}$, wild-type $\mathrm{N} 2 \mathrm{C} 3$, a syringomycin and syringopeptin double deletion $(\triangle \mathrm{SYR} \Delta \mathrm{SYP})$, and wild-type N2E2. Lowercase letters indicate statistically significant $(p<0.01,19>n>$ 29 ) differences as determined by a two-sided unpaired Student's $T$-test. Boxplots are quartile plots of the observed data. $\mathbf{f}$ Species tree showing the distribution of the island among divergent members of the Pseudomonas clade. Color coding of taxon labels summarizes the phenotypic data from $\mathbf{g}$. Colored squares indicate that an individual gene (as classified by PyParanoid) is present in a given taxon. Only 15 of the 28 genes in $\mathbf{b}$ (shown in green and teal in $\mathbf{a}$, Data S1 for details) that are unique to the pathogenic strains are shown in e. The other 13 genes (shown in purple in b) are part of larger lipopeptide biosynthesis homolog groups that are not specific to the LPQ island. $g$ Fresh weight of Arabidopsis seedlings treated with one of the 18 wild-type strains assayed in this study. Shown here are means of data from two independent experiments normalized to a $10 \mathrm{mM} \mathrm{MgSO}_{4}$ control $(10<n<$ 35). Error bars represent a $95 \%$ confidence interval for each mean using a non-parametric bootstrap of 1000 resamples 
predicted pathogenic (LPQ+) or commensal (LPQ-) lifestyles within the Pfl clade.

\section{Commensalism and pathogenesis are associated with multiple genomic features}

Because deletion of the lipopeptide clusters converts N2C3 into a strain that increases lateral root density $(\triangle \mathrm{SYR} \Delta \mathrm{SYP}$, Fig. 2c, d), we considered whether presence or absence of the LPQ island alone might be sufficient to explain divergent bacterial lifestyles or if lifestyle changes are linked to additional genomic loci. To answer this question, we identified a broader monophyletic group of 85 strains with available genomes encompassing the $P$. brassicacearum clade, as well as the sister group containing the LPQ+ pathogens $P$. corrugata and $P$. mediterranea (hereafter the " $b c m$ clade", corresponds to the $P$. corrugata subgroup in [31]). Together the $b c m$ clade corresponds to the " $P$. corrugata" subgroup identified in other Pseudomonas phylogenomic studies and shares $>97 \%$ 16S identity despite containing 8 different named species [31, 46]. This group is broadly plant-associated, with 74 of the 85 genomes (87\%) coming from strains isolated from plant tissue or rhizosphere (Table S1). Constraining our analysis to a phylogenetically narrow clade containing both pathogenic and commensal plant-associated bacteria allowed us to examine lifestyle transitions over a short evolutionary time.

To test if the pathogenicity island was correlated with the presence or absence of other elements of the variable genome, we performed a genome-wide association study (GWAS) in order to link the presence and absence of specific genes (based on PyParanoid data) with the predicted pathogenic phenotype (i.e. presence of the LPQ island). We utilized treeWAS, which is designed to account for the strong effect of population structure in bacterial datasets [47]. Using treeWAS, we identified 41 genes outside of the LPQ island, which are significantly $(p<0.01)$ associated with the presence of the island based on three independent statistical tests (Table S4). Four hundred and seven additional genes passed one or two significance tests, demonstrating that many genetic loci in the $b \mathrm{~cm}$ clade are influenced by the presence of the LPQ island in the genome.

We explored the physical locations and annotations of the loci with significant associations with the LPQ island to identify clusters of genes with cohesive functional roles in plant-microbe interactions. Beyond the LPQ island we found five additional genomic loci: two are positively correlated with the LPQ island and three are negatively correlated with the LPQ island (Fig. 3, Table S4). A subset of the genes significantly associated with the LPQ island are found in two small $(<10 \mathrm{~kb})$ genetic clusters with unknown functions (putative pathogenicity islets I and II-PPI1 and PPI2), which are correlated with the presence of the LPQ island in validated pathogenic strains as well as LPQ+ genomes that have not been tested.

The three loci that correlated with the absence of the LPQ island included a locus containing 28 genes encoding a type III secretion system (T3SS) and effectors (Table S4). This T3SS island is part of the broad Hrp family of T3SSs important for $P$. syringae virulence [2]. Despite their connotation as virulence genes, T3SSs can also be important for beneficial interactions like nodulation by rhizobia, providing a precedent for its occurrence in predicted commensal strains in the $\mathrm{bcm}$ clade [48]. The exact island identified here is found in commensal rhizosphere $\mathrm{bcm}$ clade strains Q8r1-96 and Pf29Arp where it is known as the Rop system and is necessary for the suppression of pathogen- and effector-triggered immunity (Fig. 3) [49, 50]. Moreover, the specific groups identified by PyParanoid as part of the Rop system are distinct from the Hrp genes of $P$. syringae, suggesting that T3SS genes in commensal rhizosphere bacteria have a distinct evolutionary history.

Many commensal strains in the $\mathrm{bcm}$ clade also have a single "orphaned" T3SS effector similar to the $P$. syringae hopAA gene (named ropAA in Q8r1-96) [49, 51]. Commensal strains are also highly likely to contain a gene cluster for biosynthesis of diacetylphloroglucinol (DAPG), a well-studied and potent antifungal compound important for biocontrol of phytopathogens [52]. All six genetic loci (LPQ, PPI1, PPI2, T3SS, DAPG, and hopAA, Table S5) are polyphyletic, revealing a complex evolutionary history of lifestyle transitions within the $\mathrm{bcm}$ clade (Fig. 3). Interestingly, these genomic features are largely restricted to the bcm clade, as a broader survey of the genus Pseudomonas and the Pfl clade indicates only sporadic distribution of these genes (Figure S4). Collectively, this indicates that acquisition or loss of a pathogenicity island correlates with reciprocal gain and loss of genes that may be associated with commensalism within a clade of plant-associated of bacteria.

\section{Transitions between pathogenesis and commensalism arise from homologous recombination-driven genomic variation}

To further understand the evolutionary history of the $\mathrm{bcm}$ clade, we searched for artifacts of the HGT events that might cause the polyphyletic distribution of the six lifestyleassociated loci. For example, we might expect to see evidence of HGT such as genomic islands integrated at multiple distinct genomic locations or islands with a phylogenetic history very distinct from the core genome phylogeny. Additionally, we might find evidence of specific HGT mechanisms such as tRNA insertion sites, transposons, and plasmid- or prophage-associated genes [53, 54]. We used the PyParanoid database to examine the flanking 


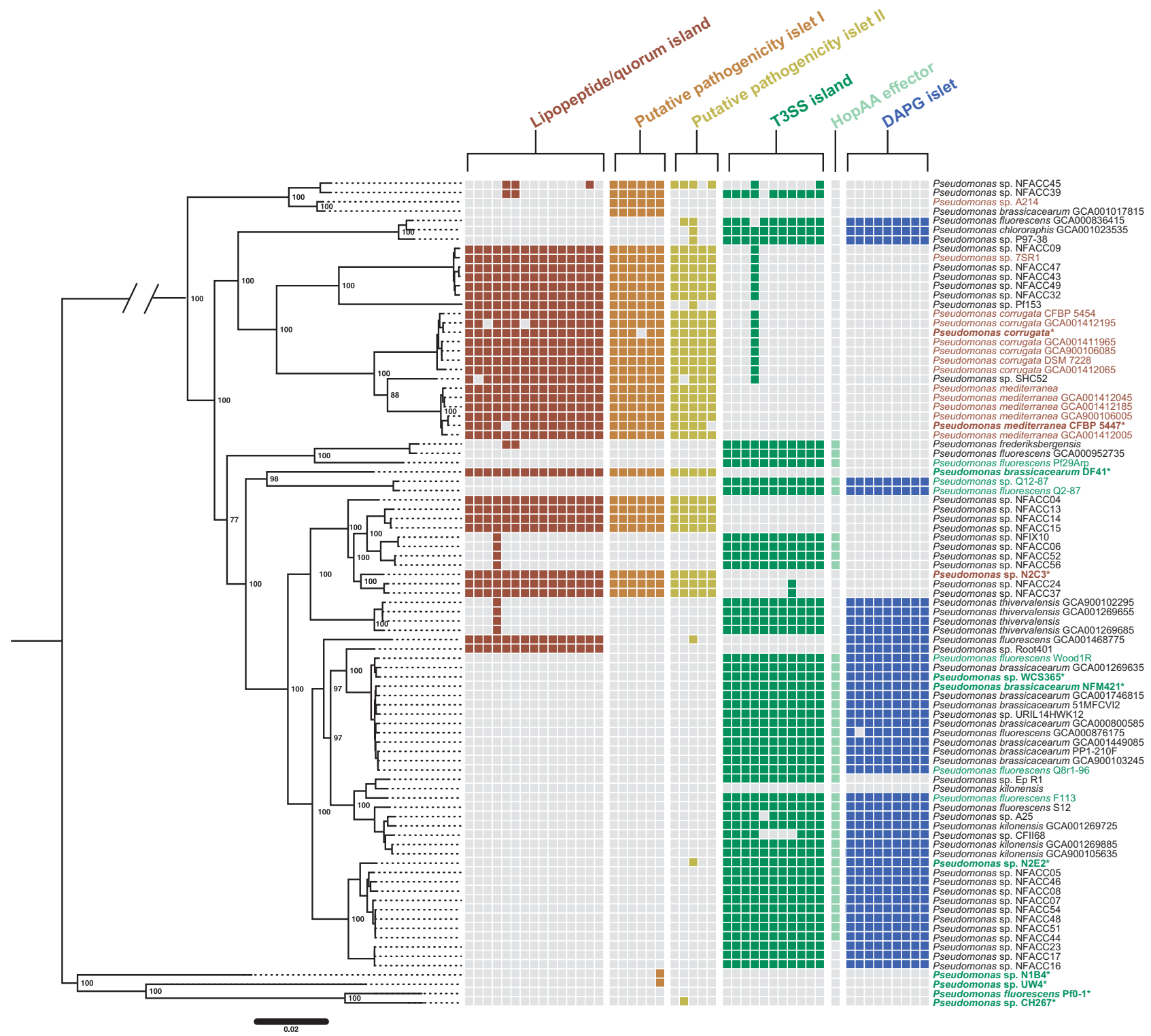

Fig. 3 Polyphyletic distribution of pathogenic and commensal genomic islands within the $\mathrm{bcm}$ clade. A species tree was built for the $\mathrm{bcm}$ clade using a concatenated alignment of 2030 conserved genes. Colorcoded squares represent presence and absence of individual genes associated with each locus based on PyParanoid presence-absence data. In the case of both the LPQ and T3SS, only a subset of genes to the island are shown because both islands contain genes that are part of larger homolog groups not specific to either island. Bold taxon names highlight strains experimentally tested for commensal (green) or pathogenic (red) behavior (Fig. $2 \mathrm{~g}$ ). For strains that we did not test experimentally, we annotated rhizosphere lifestyle based on previous reports in the literature with the same color scheme. All six loci exhibit a highly polyphyletic distribution in extant strains suggesting that the pathogenic and commensal lifestyles have a complex evolutionary history in this clade regions of each of the five islands and the hopAA gene. We detected each locus only in a single genomic context, with flanking regions conserved in all $\mathrm{bcm}$ genomes (Fig. $4 \mathrm{a}$ and Figures S5-S10). These loci are not physically linked in any of the $\mathrm{bcm}$ genomes, suggesting that linkage disequilibrium of these loci is driven by ecological selection ("eco-LD"), not physical genetic linkage (Fig. 4b and Figures S5-S10) [55]. Finally, there were no obvious genomic signatures of transposition, conjugation, transduction, or site-specific integration; all of which are commonly associated with HGT of genomic islands [54, 56]. Together, the absence of HGT signals and the conservation of the flanking regions signify homologous recombination of flanking regions as the primary mechanism driving gain or loss of the lifestyleassociated loci.

Recombination events between distantly related strains can lead to incongruencies between gene and species phylogenies. To identify recombination events leading to island 
A

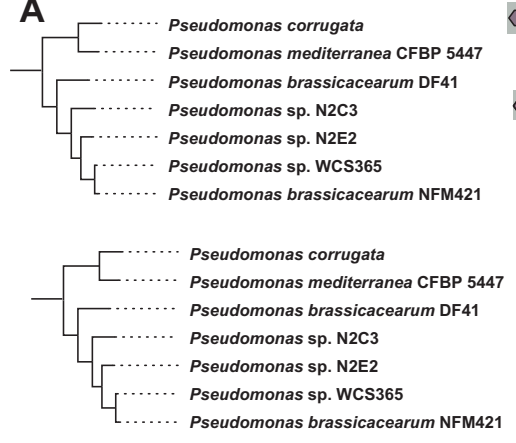

B
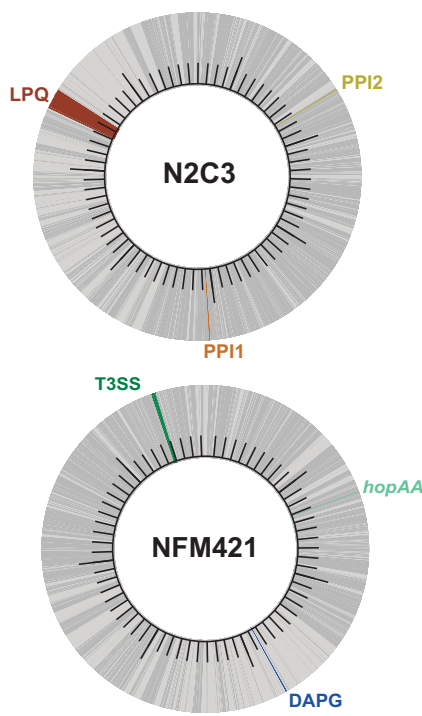

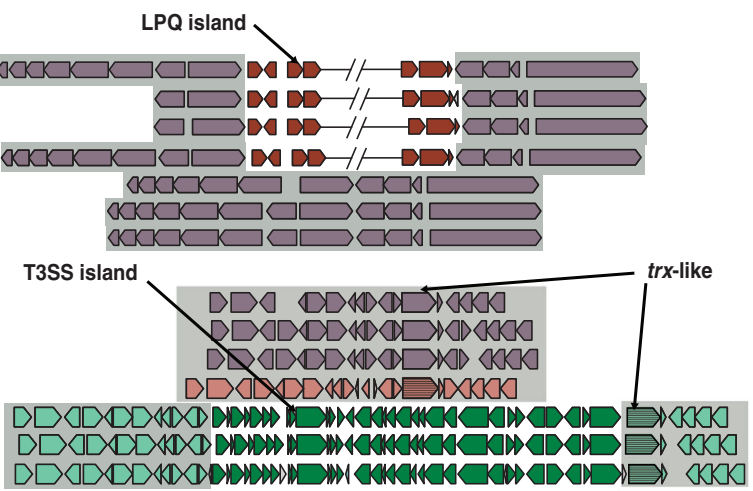

C

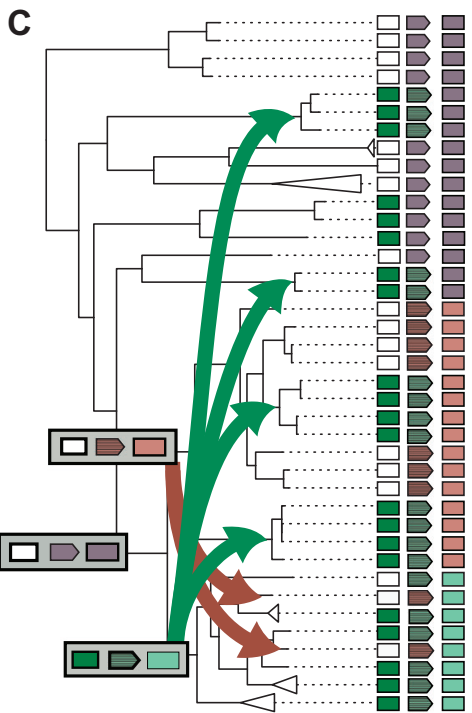

D
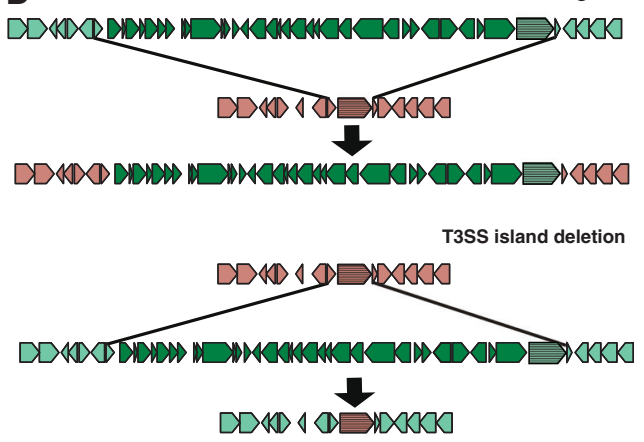

Fig. 4 Phylogenomic evidence for independent island gain and loss through homologous recombination of flanking regions. a Insertion sites for each locus are conserved throughout the $\mathrm{bcm}$ species complex. The LPQ and type III secretion system (T3SS) island insertion sites are shown here for the seven $\mathrm{bcm}$ clade strains that we characterized experimentally. All insertion sites for all six loci in the 85 strains can be found in Figures S5-S10. The tree shows the evolutionary relationship of the $\mathrm{bcm}$ strains. The gray background highlights that the conserved regions flanking both islands are adjacent in strains lacking the island. The location of the trx-like gene is shown adjacent to the T3SS, which is used as a marker for recombination in c. b Circular genome plots of two representative complete genomes from the pathogenic strain N2C3 and the beneficial strain NFM421. Darker gray regions of the genome represent the core genome of the $\mathrm{bcm}$ clade, while light gray denotes the variable genome. c A species phylogeny for the $\mathrm{bcm}$ clade adapted from Fig. 3 to show evidence of homologous recombination of the T3SS island inferred through phylogenetic incongruencies between the species tree and the phylogeny of the trxlike gene (see Figure S13). Dark green rectangles denote taxa that have the T3SS island, while white rectangles represent taxa missing the island. Based on the phylogenetic evidence, we propose a parsimonious model for evolution of the T3SS island where gain of the T3SS island led to divergence of an ancestral lineage (purple arrow/rectangle) into a lineage with the T3SS present (light green arrow/rectangle) and a lineage with the T3SS absent (light red arrow/rectangle). However, subsequent gain (green arrows) or loss (red arrows) of the T3SS island has led to phylogenetic incongruence between the adjacent $t r x$-like gene and the conserved loci used to construct the species tree (i.e. the $\mathrm{bcm}$ clade clonal phylogeny). d A diagram of the proposed homologous recombination mechanism leading to island gain (top) and loss (bottom). Both events lead to insertion of a trx-like allele with a distinct history from the surrounding conserved chromosomal loci

In contrast, the history of the T3SS island shows evidence that the island was occasionally acquired from divergent donors.

Since the T3SS island's history included several instances of recombination between distantly related donors and recipients, we reasoned that there might be signatures of such events in regions flanking the island. To test this hypothesis, we built phylogenies of conserved genes phylogenetically indistinguishable from clonal inheritance. 
flanking the T3SS. For one gene downstream of the T3SS island integration site (annotated as "trx-like", due to annotation as a thioredoxin domain-containing protein), we found that the gene tree was incongruent with the species tree, indicating HGT was prevalent in the history of the trxlike gene despite its conservation in all extant members of the $\mathrm{bcm}$ clade (Figure S13). By integrating the T3SS presence-absence data with the trx-like phylogeny and the species tree, we developed a model based on phylogenetic evidence that explains the origins of the T3SS island in extant bcm strains (Fig. 4c, d and S13). Our model implicates homologous recombination between regions flanking genomic islands as the likely mechanism behind gain and loss of lifestyle-associated loci (Fig. 4d). This provides an evolutionary mechanism underpinning the polyphyletic distribution of commensal- and pathogenic-associated islands and possibly strain lifestyle within closely related strains of Pfl.

\section{Quorum interactions drive lipopeptide production and cooperative pathogenesis}

Quorum-sensing mechanisms are generally associated with monophyletic groups and their maintenance is thought to be enforced through kin selection [57]. However, the polyphyletic distribution of the LPQ island (Fig. 4) suggests that distantly related $\mathrm{LPQ}+$ strains might cooperate to induce pathogenesis to the exclusion of more closely related LPQstrains. If the $l u x I_{L P Q} / l u x R_{L P Q}$ system allows cooperation among distantly related LPQ+ strains, we would expect the system to be phylogenetically distinct from other AHL synthases and specifically associated with lipopeptideproducing strains within Pseudomonas. We found that LuxI $_{\mathrm{LPQ}}$ represented a monophyletic clade of Pseudomonas LuxI sequences as delineated using our Pseudomonas reference pangenome (Fig. 5a). Furthermore, the presence of $\operatorname{LuxI}_{\mathrm{LPQ}}$ had a positive correlation with all of the 14 other lipopeptide genes across the entire Pseudomonas clade (Fig. 5b). While there are many lipopeptide-producing strains that lack $\operatorname{LuxI}_{\mathrm{LPQ}}$ (mostly $P$. syringae), every strain that has LuxI $\mathrm{I}_{\mathrm{LPQ}}$ also has the entire LPQ island (Table S3). These in silico results indicate that $\operatorname{LuxI}_{\mathrm{LPQ}}$ is specifically associated with cyclic lipopeptide-producing Pseudomonas spp. across the entire genus.

To test if the LuxI $\mathrm{LPQ}_{\mathrm{LP}}$ homologs share the same signaling molecule, we co-inoculated Arabidopsis seedlings with DF41 (a non-pathogenic LPQ+ strain) and N2C3 $\Delta l u x I_{L P Q}$ and $\triangle l u x R_{L P Q}$ mutants, deficient in production of the AHL signal and signal perception, respectively. We found that DF41 restored pathogenicity of the non-pathogenic $\Delta l u x$ $I_{L P Q}$ AHL synthase mutant, indicating that it can provide an activating AHL signal in trans. However, DF41 did not restore pathogenicity of the $\Delta l u x R_{L P Q}$ regulatory mutant
(Fig. 5c). Using a visual screen consisting of an AHL biosensor strain that produces the purple pigment violacein in response to short-chain AHL molecules, we found that all of the strains containing the LPQ island in the $P$. brassicacearum clade $(P$. corrugata, $P$. mediterranea, DF41, and $\mathrm{N} 2 \mathrm{C} 3)$ robustly elicited pigment production. The three fuscovaginae-like strains with the LPQ island (IRRI 6609, IRRI 7007, and S-E1) did not activate the biosensor, nor did any of the strains without the island (0 out of 20) (Fig. 5d) [20]. To confirm biosensor activity within the $\mathrm{bcm}$ clade, we utilized a quantitative assay for violacein production. We found that the four strains in the $b \mathrm{~cm}$ clade containing the LPQ island ( $P$. corrugata, $P$. mediterranea, DF41, and N2C3) increased violacein production, whereas two strains without the island (N2E2 and NFM421) and the N2C3 $\triangle \operatorname{luxI}_{L P Q}$ strain failed to induce pigment production (Fig. 5e). Reports from P. corrugata, $P$. mediterranea, $P$. brassicacearum DF41, and $P$. fluorescens in 5 specifically implicate production of a C6-AHL molecule [41, 42, 45, 58], which is a strong inducer of the violacein-producing biosensor. Thus, the LPQ island has the capability to allow polyphyletic Pseudomonas strains within the $\mathrm{bcm}$ clade to coordinate lipopeptide production and possibly pathogenesis through community C6-AHL levels.

\section{Discussion}

Here we provide evidence that homologous recombination of a large pathogenicity island is associated with the transition between commensal and pathogenic lifestyles in a clade of plant-associated Pseudomonas. By focusing on the genetic variation within a single OTU (the $\mathrm{bcm}$ clade), we found that the presence of the pathogenicity island strongly predicted the presence or absence of five additional loci. Interestingly, the same island-based adaptations appear in multiple independent lineages, providing a compelling example of convergent gene gain and loss.

To understand the processes behind the convergent evolution within this clade, we gathered three independent lines of evidence that all support homologous recombination as the dominant mechanism of genomic island variation. The absence of signatures of HGT, the conservation of the flanking regions, and the incongruency of conserved flanking genes with the species tree are all illustrative of homologous recombination. In particular, by reconciling the trx-like gene phylogeny with the species tree, we were able to identify specific instances where homologous recombination led to the gain or loss of the T3SS island (Fig. 4, S13).

The genomic and phenotypic diversity of the $\mathrm{bcm}$ clade reveals the complexity inherent in studying the rhizosphere microbiome, particular when trying to link particular $16 \mathrm{~S}$ 

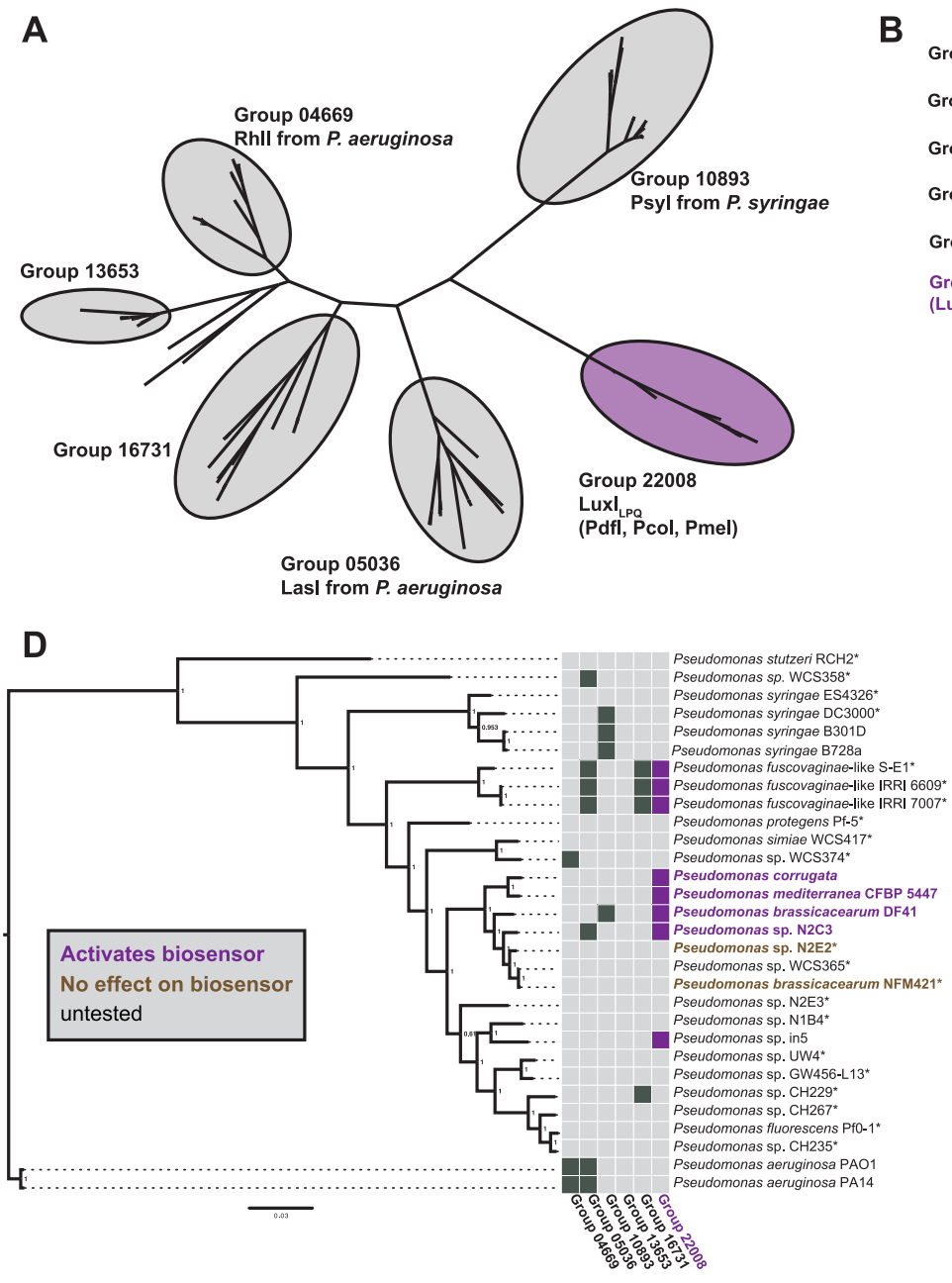

B

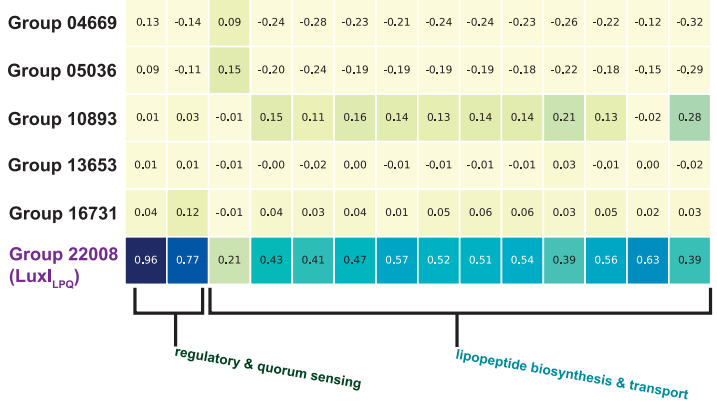

C

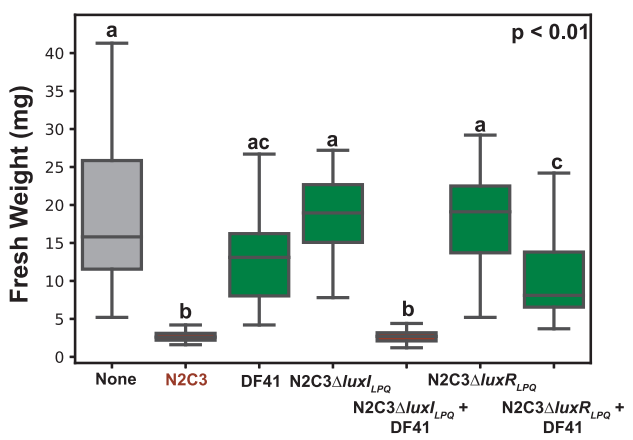

E

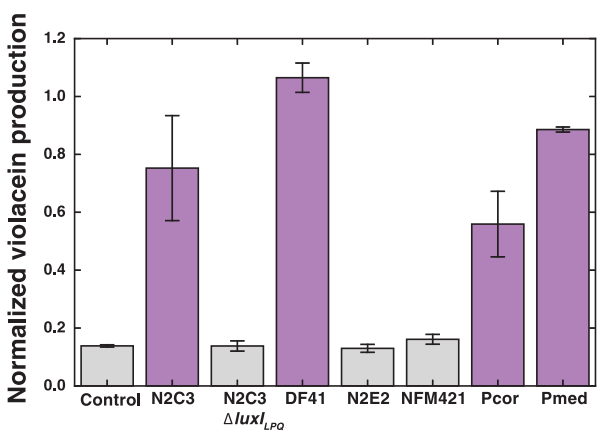

Fig. 5 A lipopeptide-associated quorum-sensing in divergent Pseudomonas spp. a Gene tree showing the phylogenetic relationship of the six acyl-homoserine lactone (AHL) synthase homology groups identified by PyParanoid. Light gray squares indicate the absence of a gene and dark gray or purple squares show the presence of a gene. Group 22008 contains the LuxI $\mathrm{I}_{\mathrm{LPQ}}$ protein sequence from N2C3 as well as protein sequences from other LPQ+ strains (PdfI, PcoI, and PmeI) [41, 42, 45]. b Correlation coefficients between each AHL synthase homology group and the 14 other LPQ island homology groups across the entire Pseudomonas clade showing that the presence of $\operatorname{LuxI}_{\mathrm{LPQ}}$ is correlated with the capacity for lipopeptide biosynthesis across the entire Pseudomonas genus. c Fresh weight of seedlings inoculated with $10 \mathrm{mM} \mathrm{MgSO}_{4}$, wild-type $\mathrm{N} 2 \mathrm{C} 3$, wild-type DF41, the AHL synthase deletion mutant $\mathrm{N} 2 \mathrm{C} 3 \Delta l u x I_{L P O}$ alone or co-inoculated with DF41, and the AHL-binding transcriptional regulator mutant $\mathrm{N} 2 \mathrm{C} 3 \triangle \operatorname{lux} R_{L P Q}$ alone or co-inoculated with DF41. Lowercase letters indicate statistically significant $(p<0.01,23>n>12)$ differences as

sequences with functions in single strains. We found that labels like "commensal" and "pathogen" break down over short evolutionary distances within a well-studied clade of Pseudomonas spp. Moreover, we found that one strain (DF41) may function as a commensal or beneficial strain in determined by a two-sided unpaired Student's $T$-test. Data shown are from one of two independent experiments performed with similar results. Boxplots are quartile plots of the observed data. d Species tree based on 217 housekeeping genes showing the distribution of six AHL synthase homology groups identified using PyParanoid. All strains with an asterisk were tested in a visual screen for induction of pigment production in the $C$. violaceum CV026 AHL biosensor strain, but only strains in the $\mathrm{bcm}$ clade with the LPQ island resulted in visible pigment production. Within the $\mathrm{bcm}$ clade, purple and brown taxon names show strains that were confirmed to induce or have no effect, respectively, on violacein production in a quantitative assay. e Barplot showing quantitative violacein production by $C$. violaceum $\mathrm{CV} 026$ by specified strains in the $\mathrm{bcm}$ clade. Data are the average of three biological replicates and error bars indicate the standard deviation $(n=3)$. Violacein production was normalized to total bacterial growth by calculating the ratio of $\mathrm{OD}_{585} / \mathrm{OD}_{764}$

isolation but might exacerbate the effects of bad actors through inter-strain quorum-sensing. The role that the various loci play in the physiology and ecology of these strains (i.e. lifestyle) is still largely unclear. Thus, we emphasize that for the majority of strains in the $b c m$ clade we are only 
predicting lifestyle; the actual effect on a plant host under different conditions must be determined empirically.

However, our core inference about lifestyle in the $\mathrm{bcm}$ clade based on the LPQ and T3SS islands relies substantially on experimentally validated evidence. The LPQ island is a known determinant of pathogenicity in the $\mathrm{bcm}$ clade [41, 42], whereas the T3SS has been found to be involved in suppressing host immunity in beneficial rhizosphere strains $[49,50]$. These two islands have a perfect anti-correlation in the $\mathrm{bcm}$ clade, with the T3SS occurring exclusively in beneficial or commensal strains, and the LPQ strains occurring only in strains described as pathogens with the exception of DF41 (Figs. 2 and 3). DF41 had no negative effect on Arabidopsis in isolation (Fig. 2), and has antifungal biocontrol activity [43], thus appearing to be a authentic commensal strain. However, DF41 can make both the AHL signal molecule and its biocontrol activity is associated with production of a lipopeptide, suggesting it contains features of both pathogens and commensals [43].

Recent reports in DF41 (as well as the LPQ+ strain $P$. fluorescens in5 and $P$. corrugata) support a more complex regulatory mechanism by implicating two additional transcriptional regulators that have LuxR DNA-binding domains (but no AHL-binding domain) [45, 58, 59]. One of these genes, $r f i A$, is essential for lipopeptide production in DF41, while quorum-sensing is not, demonstrating that the LPQ island in DF41 is regulated differently than in $\mathrm{N} 2 \mathrm{C} 3$ [45]. Identifying the signals and environmental conditions that activate lipopeptide production in different $\mathrm{LPQ}+$ strains will be crucial for further elucidating the link between this island and antifungal and plant-pathogenic strain lifestyles.

The LPQ island has genome-wide implications for strains in the $b \mathrm{~cm}$ clade, as we identified five loci in either positive or negative "eco-LD" with the LPQ island, implying epistasis and selection for one lifestyle or another. However, it is unclear how exactly microbe-mediated effects on the host might translate to microbial fitness in the rhizosphere and thus selection for the presence or absence of these loci. For example, do pathogenic strains outcompete commensal strains in a diseased plant? Furthermore, do recently diverging clades of pathogenic or commensal bcm strains even inhabit the same ecological niche? One possibility is that the LPQ island is a "nichedefining" evolutionary event that separates an incipient pathogen from its commensal predecessors, leading to further divergence [60]. Since the $\mathrm{bcm}$ clade contains pathogen to commensal transitions as well, the T3SS may have a similar niche-defining role, possibly manipulating immune responses of the host plant to favor other T3SS + strains.

More broadly, our work provides evidence that epistatic genome-wide patterns in the pangenome have strong phenotypic implications for closely related bacteria. These patterns may be difficult to identify when bacterial OTUs or populations are sparsely sampled. The recent increase of genomic data from many isolate populations makes GWAS and epistasis analyses a broadly powerful approach for identifying ecologically important loci that might not be identified using traditional genetics.

Acknowledgements We thank Dr. Clay Fuqua for providing the Chromobacterium violaceum CV026 biosensor strain, Dr. Teresa de Kievit and Dr. Ricardo Oliva for providing Pseudomonas isolates, and Dr. Adam Steinbrenner and Dr. Justin Meyer for critical reading of the manuscript. RAM is a Simons Foundation Fellow of the Life Sciences Research Foundation. This work was also supported by an NSERC Discovery Grant (NSERC-RGPIN-2016-04121), Canada Foundation for Innovation, and Canada Research Chair grants awarded to $\mathrm{CHH}$, and an NSERC USRA to SSH. The computational research was carried out with support provided by WestGrid and Compute Canada. RAM and $\mathrm{CHH}$ designed research and discussed results. RAM and SSH performed experiments. RAM wrote code and performed all bioinformatics analyses. RAM wrote the manuscript with input from $\mathrm{CHH}$ and $\mathrm{SSH}$.

\section{Compliance with ethical standards}

Conflict of interest The authors declare that they have no conflict of interest.

Publisher's note: Springer Nature remains neutral with regard to jurisdictional claims in published maps and institutional affiliations.

\section{References}

1. Hirsch A. Plant-microbe symbioses: a continuum from commensalism to parasitism. Symbiosis. 2004;37:345-63.

2. Xin X-F, Kvitko B, He SY. Pseudomonas syringae: what it takes to be a pathogen. Nat Rev Microbiol. 2018;16:316.

3. Jones KM, Kobayashi H, Davies BW, Taga ME, Walker GC. How rhizobial symbionts invade plants: the Sinorhizobium Medicago model. Nat Rev Microbiol. 2007;5:619-33.

4. Savory EA, Fuller SL, Weisberg AJ, Thomas WJ, Gordon MI, Stevens DM, et al. Evolutionary transitions between beneficial and phytopathogenic Rhodococcus challenge disease management. eLife. 2017;6:1-28.

5. Tenaillon O, Skurnik D, Picard B, Denamur E. The population genetics of commensal Escherichia coli. Nat Rev Microbiol. 2010;8:207-17.

6. Lin D, Koskella B. Friend and foe: factors influencing the movement of the bacterium Helicobacter pylori along the parasitism-mutualism continuum. Evol Appl. 2015;8:9-22.

7. Quibod IL, Grande G, Oreiro EG, Borja FN, Dossa GS, Mauleon $\mathrm{R}$, et al. Rice-infecting Pseudomonas genomes are highly accessorized and harbor multiple putative virulence mechanisms to Cause Sheath Brown Rot. PLoS ONE. 2015;10:1-25.

8. Trantas EA, Licciardello G, Almeida NF, Witek K, Strano CP, Duxbury $\mathrm{Z}$, et al. Comparative genomic analysis of multiple strains of two unusual plant pathogens: Pseudomonas corrugata and Pseudomonas mediterranea. Front Microbiol. 2015;6:1-19.

9. Haney CH, Samuel BS, Bush J, Ausubel FM. Associations with rhizosphere bacteria can confer an adaptive advantage to plants. Nat Plants. 2015;1:15051.

10. Fromin N, Achouak W, Thiéry JM, Heulin T. The genotypic diversity of Pseudomonas brassicacearum populations isolated 
from roots of Arabidopsis thaliana: influence of plant genotype. FEMS Microbiol Ecol. 2001;37:21-9.

11. Berendsen RL, van Verk MC, Stringlis IA, Zamioudis C, Tommassen J, Pieterse CMJ, et al. Unearthing the genomes of plantbeneficial Pseudomonas model strains WCS358, WCS374 and WCS417. BMC Genomics. 2015;16:1-23.

12. Sikorski J, Jahr H, Wackernagel W. The structure of a local population of phytopathogenic Pseudomonas brassicacearum from agricultural soil indicates development under purifying selection pressure. Environ Microbiol. 2001;3:176-86.

13. Belimov A, Dodd I, Safronova V, Hontzeas N, Davies W. Pseudomonas brassicacearum strain Am3 containing 1aminocyclopropane-1-carboxylate deaminase can show both pathogenic and growth-promoting properties in its interaction with tomato. J Exp Bot. 2007;58:1485-95.

14. Bulgarelli D, Rott M, Schlaeppi K, Ver Loren van Themaat E, Ahmadinejad N, Assenza F, et al. Revealing structure and assembly cues for Arabidopsis root-inhabiting bacterial microbiota. Nature. 2012;488:91-5.

15. Lundberg DS, Lebeis SL, Paredes SH, Yourstone S, Gehring J, Malfatti S, et al. Defining the core Arabidopsis thaliana root microbiome. Nature. 2012;488:86-90.

16. Haney CH, Wiesmann CL, Shapiro LR, Melnyk RA, O'Sullivan LR, Khorasani S, et al. Rhizosphere-associated Pseudomonas induce systemic resistance to herbivores at the cost of susceptibility to bacterial pathogens. Mol Ecol. 2018;27:1833-47.

17. Scarlett CM, Fletcher JT, Roberts P, Lelliott RA. Tomato pith necrosis caused by Pseudomonas corrugata n. sp. Ann Appl Biol. 1978;88:105-14.

18. Kim J, Choi O, Kim W-I. First report of sheath brown rot of rice caused by Pseudomonas fuscovaginae in Korea. Plant Dis. 2015;99:1033.

19. Mcclean KH, Winson MK, Fish L, Taylor A, Chhabra SR, Camara M, et al. Quorum sensing and Chromobacteriurn violaceum: exploitation of violacein production and inhibition for the detection of $\mathrm{N}$-acyl homoserine lactones. Microbiology. 1997;143:3703-11.

20. Latifi A, Winson MK, Foglino M, Bycroft BW, Stewart GSAB, Lazdunski A, et al. Multiple homologues of LuxR and LuxI control expression of virulence determinants and secondary metabolites through quorum sensing in Pseudomonas aeruginosa PAO1. Mol Microbiol. 1995; 17:333-43.

21. Joshi C, Patel P, Singh A, Sukhadiya J, Shah V. Frequencydependent response of Chromobacterium violaceum to sonic stimulation and altered gene expression associated with enhanced violacein production at $300 \mathrm{~Hz}$. Curr Sci. 2018;115:83-90.

22. Hmelo LR, Borlee BR, Almblad H, Love ME, Randall TE, Tseng BS, et al. Precision-engineering the Pseudomonas aeruginosa genome with two-step allelic exchange. Nat Protoc. 2015;10:1820-41.

23. Rietsch A, Vallet-Gely I, Dove SL, Mekalanos JJ. ExsE, a secreted regulator of type III secretion genes in Pseudomonas aeruginosa. Proc Natl Acad Sci USA. 2005;102:8006-11.

24. Edgar RC. Search and clustering orders of magnitude faster than BLAST. Bioinformatics. 2010;26:2460-1.

25. Geels FP, Schippers B. Selection of antagonistic fluorescent Pseudomonas spp. and their root colonization and persistence following treatment of seed potatoes. J Phytopathol. 1983;108:193-206.

26. De Weert S, Dekkers LC, Kuiper I, Bloemberg GV, Lugtenberg BJJ. Generation of enhanced competitive root-tip-colonizing Pseudomonas bacteria through accelerated evolution. J Bacteriol. 2004;186:3153-9.

27. Kamilova F, Lamers G, Lugtenberg B. Biocontrol strain Pseudomonas fluorescens WCS365 inhibits germination of Fusarium oxysporum spores in tomato root exudate as well as subsequent formation of new spores. Environ Microbiol. 2008;10:2455-61.

28. Achouak W, Sutra L, Heulin T, Meyer JM, Fromin N, Degraeve $\mathrm{S}$, et al. Pseudomonas brassicacearum sp. nov. and Pseudomonas thivervalensis sp. nov., two root-associated bacteria isolated from Brassica napus and Arabidopsis thaliana. Int $\mathrm{J}$ Syst Evol Microbiol. 2000;50:9-18.

29. Thorgersen MP, Lancaster WA, Vaccaro BJ, Poole FL, Rocha AM, Mehlhorn T, et al. Molybdenum availability is key to nitrate removal in contaminated groundwater environments. Appl Environ Microbiol. 2015;81:4976-83.

30. Price MN, Wetmore KM, Waters RJ, Callaghan M, Ray J, Liu H, et al. Mutant phenotypes for thousands of bacterial genes of unknown function. Nature. 2018;557:503-9.

31. Hesse C, Schulz F, Bull CT, Shaffer BT, Yan Q, Shapiro N, et al. Genome-based evolutionary history of Pseudomonas spp. Environ Microbiol. 2018;20:2142-59.

32. Emms DM, Kelly S. OrthoFinder: solving fundamental biases in whole genome comparisons dramatically improves orthogroup inference accuracy. Genome Biol. 2015;16:1-14.

33. Emms DM, Kelly S. OrthoFinder2: fast and accurate phylogenomic orthology analysis from gene sequences. bioRxiv. 2018;466201.

34. Papenfort K, Bassler BL. Quorum sensing signal-response systems in Gram-negative bacteria. Nat Rev Microbiol. 2016;14:57688.

35. Scholz-Schroeder BK, Hutchison ML, Grgurina I, Gross DC. The contribution of syringopeptin and syringomycin to virulence of Pseudomonas syringae pv. syringae strain B301D on the basis of sypA and syrB1 biosynthesis mutant analysis. Mol Plant Microbe Interact. 2001;14:336-48.

36. Hutchison ML, Gross DC. Lipopeptide phytotoxins produced by Pseudomonas syringae pv. syringae: comparison of the biosurfactant and ion channel-forming activities of syringopeptin and syringomycin. Mol Plant Microbe Interact. 1997;10:347-54.

37. Baltrus DA, Nishimura MT, Romanchuk A, Chang JH, Mukhtar MS, Cherkis K, et al. Dynamic evolution of pathogenicity revealed by sequencing and comparative genomics of 19 Pseudomonas syringae isolates. PLoS Pathog. 2011;7:e1002132.

38. Hockett KL, Nishimura MT, Karlsrud E, Dougherty K, Baltrus DA. Pseudomonas syringae CC1557: a highly virulent strain with an unusually small type III effector repertoire that includes a novel effector. Mol Plant Microbe Interact. 2014;27:923-32.

39. Strano CP, Bella P, Licciardello G, Fiore A, Lo Piero AR, Fogliano V, et al. Pseudomonas corrugata crpCDE is part of the cyclic lipopeptide corpeptin biosynthetic gene cluster and is involved in bacterial virulence in tomato and in hypersensitive response in Nicotiana benthamiana. Mol Plant Pathol. 2015;16:495-506.

40. Patel HK, Matiuzzo M, Bertani I, De Paul Bigirimana V, Ash GJ, Höfte $\mathrm{M}$, et al. Identification of virulence associated loci in the emerging broad host range plant pathogen Pseudomonas fuscovaginae. BMC Microbiol. 2014;14:1-13.

41. Licciardello G, Strano CP, Bertani I, Bella P, Fiore A, Fogliano V, et al. N-acyl-homoserine-lactone quorum sensing in tomato phytopathogenic Pseudomonas spp. is involved in the regulation of lipodepsipeptide production. J Biotechnol. 2012;159:274-82.

42. Licciardello G, Bertani I, Steindler L, Bella P, Venturi V, Catara V. Pseudomonas corrugata contains a conserved N-acyl homoserine lactone quorum sensing system; its role in tomato pathogenicity and tobacco hypersensitivity response. FEMS Microbiol Ecol. 2007;61:222-34.

43. Berry C, Fernando WGD, Loewen PC, de Kievit TR. Lipopeptides are essential for Pseudomonas sp. DF41 biocontrol of Sclerotinia sclerotiorum. Biol Control. 2010;55:211-8. 
44. Michelsen CF, Watrous J, Glaring MA, Kersten R, Koyama N, Dorrestein PC. Nonribosomal peptides, key biocontrol components for Pseudomonas fluorescens In5, isolated from a greenlandic suppressive soil. mBio. 2015;6:1-9.

45. Berry CL, Nandi M, Manuel J, Brassinga AKC, Fernando WGD, Loewen PC, et al. Characterization of the Pseudomonas sp. DF41 quorum sensing locus and its role in fungal antagonism. Biol Control. 2014;69:82-9.

46. Garrido-Sanz D, Meier-Kolthoff JP, Göker M, Martín M, Rivilla $\mathrm{R}$, Redondo-Nieto M. Genomic and genetic diversity within the Pseudomonas fluorescens complex. PLoS ONE. 2016;11: e0150183.

47. Collins C, Didelot X. A phylogenetic method to perform genomewide association studies in microbes that accounts for population structure and recombination. PLoS Comput Biol. 2018;14:1-21.

48. Krause A, Doerfel A, Göttfert M. Mutational and transcriptional analysis of the type III secretion system of Bradyrhizobium japonicum. Mol Plant Microbe Interact. 2002;15:1228-35.

49. Mavrodi DV, Joe A, Mavrodi OV, Hassan KA, Weller DM, Paulsen IT, et al. Structural and functional analysis of the type III secretion system from Pseudomonas fluorescens Q8r1-96. J Bacteriol. 2011;193:177-89.

50. Marchi M, Boutin M, Gazengel K, Rispe C, Gauthier J-P, Guillerm-Erckelboudt A-Y, et al. Genomic analysis of the biocontrol strain Pseudomonas fluorescens Pf29Arp with evidence of T3SS and T6SS gene expression on plant roots. Environ Microbiol Rep. 2013;5:393-403.

51. Munkvold KR, Russell AB, Kvitko BH, Collmer A. Pseudomonas syringae pv. tomato DC3000 type III effector HopAA1-1 functions redundantly with chlorosis-promoting factor PSPTO4723 to produce bacterial speck lesions in host tomato. Mol Plant Microbe Interact. 2009;22:1341-55.
52. Weller DM, Landa BB, Mavrodi OV, Schroeder KL, De La Fuente L, Blouin Bankhead S, et al. Role of 2,4-diacetylphloroglucinol-producing fluorescent Pseudomonas spp. in the defense of plant roots. Plant Biol. 2007;9:4-20.

53. Clark IC, Melnyk RA, Engelbrektson A, Coates JD. Structure and evolution of chlorate reduction composite transposons. mBio. 2013;4:e00379-13.

54. Melnyk RA, Coates JD. The perchlorate reduction genomic island: mechanisms and pathways of evolution by horizontal gene transfer. BMC Genomics. 2015;16:862.

55. Cui Y, Yang X, Didelot X, Guo C, Li D, Yan Y, et al. Epidemic clones, oceanic gene pools, and eco-LD in the free living marine pathogen Vibrio parahaemolyticus. Mol Biol Evol. 2015;32:1396-410.

56. Juhas $M$, Van Der Meer JR, Gaillard M, Harding RM, Hood DW, Crook DW. Genomic islands: tools of bacterial horizontal gene transfer and evolution. FEMS Microbiol Rev. 2009;33:376-93.

57. Whiteley M, Diggle SP, Greenberg EP. Progress in and promise of bacterial quorum sensing research. Nature. 2017;551:313-20.

58. Hennessy RC, Phippen CBW, Nielsen KF, Olsson S, Stougaard P. Biosynthesis of the antimicrobial cyclic lipopeptides nunamycin and nunapeptin by Pseudomonas fluorescens strain In5 is regulated by the LuxR-type transcriptional regulator NunF. MicrobiologyOpen. 2017;6:1-14.

59. Licciardello G, Caruso A, Bella P, Gheleri R, Strano CP, Anzalone A, et al. The LuxR regulators PcoR and RfiA co-regulate antimicrobial peptide and alginate production in Pseudomonas corrugata. Front Microbiol. 2018;9:521.

60. Wiedenbeck J, Cohan FM. Origins of bacterial diversity through horizontal genetic transfer and adaptation to new ecological niches. FEMS Microbiol Rev. 2011;35:957-76. 